auch rhombisches Benzophenon, längere Zeit gekocht, wird nicht wieder fest und giebt wahrscheinlich bei geeigneten Bedingungen monokline Krystalle. Aber auch die Bildung durch Oxydation hat scheinbar Einflufs, wenigstens erhielt ich aus Diphenylessigsãure beim Kochen mit chromsaurem Kali und verdunnter Schwefelsäure nach dem Erkalten Krystalle von monoklinem Benzophenon.

Das Verhalten des Kohlenwasserstoffs bei der Oxydation bestätigt also die aus seiner Bildung hergeleitete Formel $\mathrm{C}_{6} \mathrm{H}_{5}-\mathrm{CH}_{2}-\mathrm{C}_{6} \mathrm{H}_{5}$. Er mufs als Benzylbenzol oder auch als Diphenylmethan bezeichnet werden. Ohne Frage ist derselbe identisch mit dem von Jena *) als Diphenylsumpfgas bezeichneten Product, welches durch trockene Destillation von diphenylessigsaurem Baryt erhalten wurde, und für welches Jena, wie schon der Name sagt, dieselbe Constitution annimmt.

Bonn, August 1871.

\title{
Ueber ein neues Thalliumdoppelsalz;
} von R. J. Friswell**).

Man kennt allgemein die Neigung der Platinocyanide zur Bildung von Doppelsalzen; die folgenden Experinente zeigen, dafs diese Neigung sich in einer bisher nicht beachteten Richtung entwickeln läfst.

Um das Platinocyanid des Thalliums darzustellen, vermischte ich Lösungen von Thalliumcarbonat und Platincyan-

*) Diese Annalen CLV, 86.

**) Journal of the Chemical Sosiety [2] IX, 461. 
kalium. Die Lösungen waren beide sehr heifs, da Thalliumcarbonat in kaltem Wasser nur wenig löslich ist und eine heifse Lösung es auszuscheiden beginnt, sobald sie sich nur ein wenig abkühlt.

Die Mischung der Lösungen wurde in einem bedeckten Becherglas der langsamen Abkühlung überlassen; nach kurzer Zeit, während die Lösung noch sehr heifs war, begannen prachtvoll bronzegrüne dichroïstische Krystalle in Klumpen und Büscheln von Seiten und Boden des Becherglases und von der Oberfläche der Flüssigkeit aus anzuschiefsen. Ich sammelte dieselben mit möglichst geringer Verletzung, indem ich sie durch sanftes Schütteln ablöste und dann das Ganze auf ein Filter gofs. Die Mutterlauge wurde entfernt durch leichtes Waschen mit ganz kaltem destillirtem Wasser, in welchem die Krystalle sich fast unlöslich erwiesen. (Bei späteren Darstellungen fand ich es zweckmäfsig, diefs zu unterlassen, da es den Glanz der Krystalle verdarb.) Die Krystalle wurden bei $100^{\circ}$ getrocknet. Sie sind im durchfallenden Licht prachtvoll carmosinroth, während ihre reflectirte Farbe ein intensives Bronzegrün von stark metallischem Glanz ist. So lebhaft erscheint diese letztere Farhe im Tageslicht, dafs ein Freund, dem ich das Präparat zeigte, dasselbe für Bronzefeilspähne hielt.

Anfänglich hielt ich dieses Salz für das gesuchte Thalliumplatinocyanid, ich fand jedoch bald, dafs diefs nicht der Fall war, da das Salz bei Behandlung mit Säuren stark aufbraust und viel Kohlensäure entwickelt; das Brausen hält beträchtliche Zeit an und es bleibt ein blafsrother Rückstand. Thalliumplatinocyanid dagegen ist gelblichweils und ohne allen Dichroïsmus. Man kann dasselbe darstellen, indem man Baryumplatinocyanid mit der äquivalenten Menge Thalliumsulfat zersetzt und die vom Baryumsulfat abfiltrirte Flüssigkeit zur Krystallisation bringt. Da mithin das obige Salz augenschein- 
lich nicht das gesuchte Thalliumplatinocyanid sein konnte, schritt jch zur Analyse desselben.

Die Verbrennung wurde mit granulirtem Kupferoxyd bei vorgelegtem metallischem Kupfer ausgeführt in luftleeren Rohr unter Anwendung der Sprengel'schen Luftpumpe, wie es Frankland und Armstrong ${ }^{*}$ ) beschrieben. Die Gase wurden abgepumpt und analysirt.

So wurden erhalten bei Anwendung von 0,2991 Grm. Substanz 5,089 pC. Koblenstoff und 4,697 pC. Stickstoff. Wiederbolte Bestimmungen des Stickstoffs ergaben 4,607, 4,679, 4,586 pC.; der Kohlenstoff kam ein wenig böher, im Mittel von fünf Analysen zu 5,204 pC.

Das Thallium wurde durch Jodkalium aus der durch Ammoniak alkalisch gemachten Lösung (wodurch das Jodid sehr unlöslich wird) gefällt und als TlJ bestimmt.

$0,3452 \mathrm{Grm}$. gaben $0,3890 \mathrm{TlJ}$, entsprechend $0,2395 \mathrm{Tl}$ oder $69,37 \mathrm{pC}$. $0,4965 \mathrm{Grm}$. gaben 0,5596 TlJ, entsprechend 0,3448 Tl oder $69,24 \mathrm{pC}$, Mittel $69,305 \mathrm{pC}$.

Das Platin wurde bestimmt durch Schmelzen des Salzes mit saurem schwefelsaurem Kali; hierdurch wird Thallium in Sulfat verwandelt, Cyan und Kohlensäure werden zersetzt und ausgetrieben, das Platin hinterbleibt als Metall.

0,2635 Grm. gaben 0,0446 Pt oder 16,88 pC.

Nach den erhaltenen Zahlen ist das Salz eine Verbindung von einem Molecul Thalliumcarbonat mit einem Molecul Thalliumplatinocyanid oder $\mathrm{PtT}_{2} \mathrm{Cy}_{4} \mathrm{Co}(\mathrm{TlO})_{2}$; das Moleculargewicht dieser Verbindung ist 1177,4 und die Berechnung ergiebt folgende Zusammenselzung. In 100 Theilen :

\begin{tabular}{lcc} 
& berechnet & gefunden (im Mittel) \\
$\mathrm{C}_{5}$ & 5,10 & 5,20 \\
$\mathrm{~N}_{4}$ & 4,76 & 4,66 \\
$\mathrm{O}_{8}$ & 4,08 & 3,96 (aus dem Verlust) \\
$\mathrm{Pt}$ & 16,76 & 16,88 \\
$\mathrm{Tl}_{4}$ & 69,30 & 69,30 \\
\cline { 2 - 3 } & 100,00 & $100,00 \mathrm{Tl}=204,0, \mathrm{Pt}=197,4$.
\end{tabular}

Mit warmer Salpetersäure entwickelte das Salz unter Aufbrausen Kohlensäure und liês einen rothen Rückstand,

*) Journal of the Chemical Society [2] VI, 90. 
der nach sorgfältigem Auswaschen mit warmem Wasser in siedendem Wasser gelöst, beim Erkalten in langen farblosen Nadeln auskrystallisirte.

0,3242 Grm. wurden wie oben verbrannt und gaben 6,785 pC. Kohlenstoff und 7,916 pC. Stickstoff; diese Zahlen stimmen mit der Zusammensetzung des Thalliumplatinocyanids, welches verlangt 6,76 pC. Kohlenstoff und 7,86 pC. Stickstoff.

Das Salz wird erhalten durch Vermischen von $4,334 \mathrm{Grm}$. Platincyankalium $\mathrm{P}_{2} \mathrm{CY}_{4}, 3\left(\mathrm{OH}_{2}\right)$ mit $9,360 \mathrm{Grm}$. Thalliumcarbonat, wobei folgende Reaction statthat :

$\mathrm{PtK}_{2} \mathrm{Cy}_{4}+2 \mathrm{CO}(\mathrm{TlO})_{2}=\mathrm{PtTl}_{2} \mathrm{Cy}_{4}, \mathrm{CO}(\mathrm{TlO})_{2}+\mathrm{CO}(\mathrm{KO})_{2}$.

Durch Jodkalium wird das Salz in folgender Weise zersetzt: $\mathrm{PtTl}_{2} \mathrm{Cy}_{4} \mathrm{CO}(\mathrm{TlO})_{2}+4 \mathrm{KJ}=4 \mathrm{TlJ}+\mathrm{PtK}_{2} \mathrm{Cy}_{4}+\mathrm{CO}(\mathrm{KO})_{2}$.

Man kann das Salz auch darstellen durch Vermischen von 7,094 Grm. Platincyanthallium mit 4,680 Grm. Thalliumcarbonat, wobei einfache Verbindung eintritt. Auch durch Vermischen von $4,384 \mathrm{Grm}$. Platincyanbaryum mit 9,360 Grm. Thalliumcarbonat kann dasselbe erhalten werden, gemäfs der Gleichung :

$$
\mathrm{PtBaCy}_{4}+2 \mathrm{CO}(\mathrm{TlO})_{2}=\mathrm{PtTl}_{2} \mathrm{Cy}_{4} \mathrm{CO}(\mathrm{TlO})_{2}+\mathrm{COBaO}_{2} \text {. }
$$

Die Bildung aus Platincyankalium ist sehr sonderbar und ist eine empfindliche Reaction; denn ein Krystall von Thalliumcarbonat färbt sich sofort roth, wenn er mit einer Lösung von Platincyankalium befeuchtet wird.

Das Salz ist fast unlöslich in kaltem Wasser und löst sich nur schwierig in heifsem. Die wassserige Lösung ist absolut farblus; sie scheidet beim Erkalten das Salz nicht wieder ab, wenn sie nicht sehr concentrirt ist; sie setzt jedoch weifse Krystalle ab, die entweder ein Hydrat oder blofs eine Mischung der beiden gleichzeitig abgeschiedenen Bestandtheile sind; ich habe diefs noch nicht entschieden, doch neige ich stark zu der letzteren Ansicht.

Zum Umkrystallisiren des Salzes bedient man sich am Besten einer heifsen wässerigen Lösung von Thalliumcarbonat, 
welche so verdünnt ist, dafs sie beim Erkalten nichts absetzt. Ich habe die wässerige Lòsung mil dem Spectroscop untersucht und keine Absorptionsbänder gefunden, obwohl ich wegen der tiefen Färbung der Krystalle solche zu finden erwartete.

Bei sorgfältiger Untersuchung einiger besonders grofser und reiner Krystalle unter dem Mikroscop fand ich, dafs dieselben rechtwinkelige Prismen sind; zufällig erhielt ich auch einige Krystalle, welche mit blofsem Auge gesehen in eine Spitze zu endigen schienen. Die Vergröfserung liefs leicht ersehen, dafs dieselben aus einer Anzahl stufenförmig aufeinander gesetzter Würfel bestehen und in einen einzigen kleinen Würfel endigen. Die Krystalle sind ohne Wirkung auf das polarisirle Licht. Ihre Krystallform ist somit total verschieden von derjenigen des Thalliumcarbonats und des Thalliumplatinocyanids, welche beide dem rhombischen System angehören.

Dieser völlige Wechsel der Krystallgestalt macht es wahrscheinlich, dafs das Salz nicht ein eigentliches Doppelsalz, d. h. eine blofse moleculare Vereinigung der beiden Componenten, sondern einen Körper von complicirterer Natur vorstellt.

Ueber die Einwirkung der Schwefelsäure aut die natürlichen Alkaloïde;

$$
\text { von H. E. Armstrong*). }
$$

Die Einwirkung der Schwefelsãure auf die natürlichen Alkaloïde wurde zuerst von A rppe untersucht, der $\mathbf{1 8 4 5}$

*) Journal of the Chemical Society [2] IX, 56. Die Versuche bilden einen Theil der in Gemeinschaft mit dem verstorbenen Dr. Matthiessen begonnenen Reihe von Untersuchungen. 